\title{
Cathelicidin impact on inflammatory cells
}

\author{
JUSTYNA AGIER, MAGDALENA EFENBERGER, EWA BRZEZIŃSKA-BŁASZCZYK
}

Department of Experimental Immunology, Medical University of Lodz, Lodz, Poland

\begin{abstract}
Cathelicidins, like other antimicrobial peptides, exhibit direct antimicrobial activities against a broad spectrum of microbes, including both Gram-positive and Gram-negative bacteria, enveloped viruses, and fungi. These host-derived peptides kill the invaded pathogens by perturbing their cell membranes and can neutralize biological activities of endotoxin. Nowadays, more and more data indicate that these peptides, in addition to their antimicrobial properties, possess various immunomodulatory activities. Cathelicidins have the potential to influence and modulate, both directly and indirectly, the activity of various cell populations involved in inflammatory processes and in host defense against invading pathogens. They induce migration of neutrophils, monocytes/macrophages, eosinophils, and mast cells and prolong the lifespan of neutrophils. These peptides directly activate inflammatory cells to production and release of different pro-inflammatory and immunoregulatory mediators, cytokines, and chemokines, however cathelicidins might mediate the generation of anti-inflammatory cytokines as well. Cathelicidins also modulate epithelial cell/keratinocyte responses to infecting pathogens. What is more, they affect activity of monocytes, dendritic cells, keratinocytes, or epithelial cells acting in synergy with cytokines or $\beta$-defensins. In addition, these peptides indirectly balance TLR-mediated responses of monocytes, macrophages, dendritic cells, epithelial cells, and keratinocytes. This review discusses the role and significance of cathelicidins in inflammation and innate immunity against pathogens.
\end{abstract}

Key words: cathelicidins, inflammation, innate immunity, neutrophils, monocytes, macrophages, mast cells.

(Centr Eur J Immunol 2015; 40 (2): 225-235)

\section{Introduction}

The primary function of the immune system is effective protection against various types of infectious agents from the external environment. In the course of evolution three lines of defense against invading pathogens developed. The first line includes anatomical and physiological barriers that protect the inner layers of the body. Crucial natural barriers are epithelial surfaces composed of tightly joined cells and mucous membranes. Most epithelial surfaces are associated with physiological microflora. This natural microbial population competes with pathogens for nutrients and produces antibacterial substances to inhibit pathogen growth. The protective role for the respiratory system is played by cough and sneeze reflexes, movement of cilia and pulmonary surfactant. In turn, the digestive system is protected by intestinal motility, presence of glycocalyx on the surface of epithelial cells, including enterocytes, and the low $\mathrm{pH}$ of the stomach. The gastric juice, vaginal secretions and urine, which are acidic fluids, have an additional protective function. Equally important are humoral factors present in secretions and body fluids. This group includes proteolytic enzymes, acute phase pro- teins, interferons, immunoglobulins and also antimicrobial peptides.

Defense mechanisms are mainly focused on the fast identification and efficient elimination of pathogens. The early immune response is associated with activation of the innate immunity mechanisms. Innate immunity is mediated by several humoral components (complement proteins, some cytokines) and cellular mechanisms involving neutrophils, monocytes, macrophages, mast cells, eosinophils, dendritic cells, and NK cells. Finally, the third line of defense against pathogenic invasion is the adaptive immune response which involves expansion of the lymphocyte subpopulations and production of antibodies.

In the first steps of host defense against pathogens, antimicrobial peptides (AMPs) play an important role. These peptides, also called natural antibiotics or cationic host defense peptides (CHDPs), constitute a group of relatively small (12-50 amino acid residues) molecules. AMPs are found in both prokaryotes and eukaryotes and are produced by bacteria, plants, invertebrates, and vertebrates. To date, 2544 AMPs have been reported (based on the Antimicrobial Peptide Database). These small molecules have a positive charge due to the presence of arginine and lysine residues and amphipathic structure. Amino acids

Correspondence: Ewa Brzezińska-Błaszczyk, Department of Experimental Immunology, Medical University of Lodz, Pomorska 251, 92-213 Lodz, Poland, tel./fax +48 4267573 06, e-mail: ewab@csk.umed.lodz.pl 
with hydrophobic side chain constitute about $40-50 \%$ of the structure. AMPs are able to inactivate infectious agents by forming pores in the cell membrane. This is possible due to reaction with negatively charged phospholipids of microbial membrane. CHDPs are able to kill microbes such as bacteria, viruses, fungi or protozoa in a very short time [1-3].

Various groups of organisms produce AMPs with different profiles. In mammals, two major groups of AMPs are defensins and cathelicidins. Defensins are classified into three subgroups: $\alpha, \beta$ and $\theta$. The $\alpha$-defensin family has been identified in humans, monkeys, pigs, and several rodent species. So far, six distinct $\alpha$-defensins have been found in humans. Four of these were initially isolated from neutrophils, and therefore they are called human neutrophil peptides (HNP-1, HNP-2, HNP-3, and HNP-4). These peptides are also abundant in certain macrophage populations and NK cells. The human $\alpha$-defensins HD-5 and HD-6 are expressed mainly in Paneth cells of the small intestine. The $\beta$-defensin family is widely expressed in mammals and birds. Moreover, close $\beta$-defensin homologs were found in snakes, platypus and sea anemones. In humans, $\beta$-defensins (HBD-1, HBD-2, HBD-3) are produced mainly by epithelial cells and keratinocytes. $\theta$-defensin family is found in the leukocytes of rhesus macaques [3, 4].

Cathelicidins have been isolated from many mammalian species including rabbits, horses, pigs, rats, monkeys, cattle and humans. These peptides are characterized by a great variety of structure and molecular weight. The genes responsible for synthesis of cathelicidins are approximately $2 \mathrm{~kb}$ and show four-exon/three-intron organization. Cathelicidins are synthesized as inactive precursors. The structure of precursor includes a highly conserved $\mathrm{N}$-terminal segment, cathelin domain and a $C$-terminal domain with a varied sequence and length. The $N$-terminal domain is composed of 29-30 amino acid residues and supports the release of biologically active peptides. The cathelin domain is composed of 98-114 amino acid residues but its function has not been thoroughly examined. The $C$-terminal peptide (12-100 amino acid residues), as a mature peptide, exhibits broad antimicrobial activity against both Gram-positive and Gram-negative bacteria, viruses and fungi. These peptides are produced by neutrophils, epithelial cells, keratinocytes, macrophages, mast cells, NK cells, dendritic cells, and lymphocytes [5, 6].

The only known member of the cathelicidin family expressed in humans is LL-37 (leucine-leucine-37) [7]. The human cathelicidin was identified in 1995 and first isolated from neutrophils. This cationic molecule is a linear 37-amino acid-long peptide generated by cleavage of the $C$-terminus end of the hCAP18 (human cationic antimicrobial peptide 18) precursor protein. The single gene encoding LL-37 is located on chromosome 3. The name hCAP18 denotes the length of polypeptide (18 kDa) and the cationic character of the structure. In turn, LL-37 refers to the number of residues with Leu-Leu at the $\mathrm{N}$-terminus. LL-37 and its precursor were found in different cells, tissues and body fluids. The human cathelicidin is produced mainly by neutrophils, monocytes, NK cells, mast cells, $\mathrm{B}$ cells and also colon enterocytes, epithelial cells and keratinocytes. The molecule was detected in human wound fluid, seminal plasma, vernix and tracheal aspirates of newborns [3]. Cathelicidins may be produced constitutively or synthesized in response to the presence of bacteria or their products. The expression of LL-37 can be also regulated by various endogenous factors such as proinflammatory cytokines, growth factors and the active form of vitamin D [7].

Cathelicidins exhibit a broad spectrum of antimicrobial activity against Gram-positive and Gram-negative bacteria $[1,7]$, enveloped viruses [2], and fungi [8]. It is commonly known that cathelicidins interact with the negatively charged bacterial and fungal membranes and induce membrane disruption. The most accepted mechanisms of cathelicidin action are the "carpet" model, the "barrel-stave" model, and the "toroidal pore" model [9, 10]. According to the "carpet" model, peptides are set parallel to the cell surface but do not intercalate into it. The pressure from the molecules is so strong that it causes a disruption of the cell membrane. In the "barrel-stave" mechanism, peptides are incorporated perpendicularly at the membrane creating the "barrel" with the peptides being the "staves". Non-polar components of proteins interact with membrane lipids and polar parts form the pores in the cell membrane. Finally, the "toroidal pore" theory describes the formation of pores in the cell membrane where the hydrophilic components interact with the residues of phospholipids, while the hydrophobic parts with the lipids. The effect of all mechanisms is the disintegration of the cell membrane and as a result, death of the pathogen. Additionally, cathelicidins may penetrate into the cell to bind or to degrade some molecules which are crucial to cell living. Furthermore, these peptides participate in neutralization of bacterial endotoxin, i.e. lipopolysaccharide (LPS), by blocking the interaction of the lipoglycan with LPS binding protein (LBP) [11]. Nowadays, however, more and more data indicate that apart from killing pathogens directly or indirectly, cathelicidins have a number of immunomodulatory functions that might be involved in the clearance of infection. Thus, considering the role of inflammation in host defense against pathogens it is extremely interesting to know and understand the influence of cathelicidins on inflammatory cell activities.

\section{Cathelicidins affect neutrophil activity}

Neutrophils are the most abundant type of white blood cells and it is absolutely proven that they are the first leukocytes rapidly and efficiently recruited to the site of injury and inflammation. These cells are capable of express- 
ing and/or producing various potent mediators, including numerous cytokines, chemokines, colony-stimulating factors, angiogenic factors and fibrogenic factors. What is more, neutrophils express a lot of enzymes stored in different types of cytoplasmic granules. It should be stressed that neutrophils also produce four types of $\alpha$-defensins, i.e. HNP-1, HNP-2, HNP-3, and HNP-4, accumulated in a subset of azurophilic granules. Moreover, neutrophils are the source of arachidonic acid metabolites, i.e. prostaglandins (PGs) and leukotrienes (LTs). Finally, it is well known that neutrophil granulocytes are able to generate reactive oxygen species (ROS). All these neutrophil-derived mediators influence extracellular matrix (ECM) components and strongly affect the surrounding cell activity, thereby neutrophils play a crucial role in acute and chronic inflammation. In addition, neutrophil-derived mediators have a huge impact on immune cells functioning and consequently these cells participate in the course of innate and adaptive immunity. It should be emphasized that neutrophils play a key role in host defense against viral, bacterial and fungal infections [12-14].

The average circulatory lifespan of human neutrophils is relatively short (5.4 days) and ends by spontaneous apoptosis [15]. Apoptotic neutrophils are phagocytosed without release of mediators, leading to the restriction of inflammation and tissue damage. It should be noted that some host- and pathogen-derived substances may inhibit spontaneous neutrophil apoptosis. The suppressed neutrophil apoptosis results in uncontrolled release of a variety of cytotoxic metabolites and pro-inflammatory mediators, which in turn leads to the enhancing of inflammatory response and tissue injury. Considering these data, observations that cathelicidins may influence neutrophil lifetime are of great importance. Nagaoka et al. [16, 17] clearly demonstrated that LL-37 prolongs the lifespan of neutrophils by inhibition of spontaneous apoptosis. Inhibition of programmed cell death occurs through the increased expression of anti-apoptotic protein $\mathrm{Bcl}-\mathrm{X}_{\mathrm{L}}$ and by blocking the activation of caspase-3, a key executor for apoptosis. Of importance, human cathelicidin LL-37 elongates neutrophil lifespan in vitro over the concentration range from $10 \mathrm{ng} / \mathrm{ml}$ to $5 \mu \mathrm{g} / \mathrm{ml}$. Interestingly, LL-37 induces suppression of neutrophil apoptosis acting via the activation of formyl peptide receptor (FPR) 1 and purinergic receptor $\mathrm{P}^{2} \mathrm{X}_{7}[17,18]$. Cathelicidin LL-37 can also up-regulate the expression of $\mathrm{P} 2 \mathrm{X}_{7}$ receptors on these cells [19].

It was also documented that in vitro LL-37 induces neutrophil migration and chemotaxis and this effect is mediated via FPR1 molecules [19-22]. What is more, dose-dependent neutrophil migration in response to murine cathelicidin-related antimicrobial peptide (CRAMP)-stimulation was observed [23]. Porcine proline-arginine (PR)rich antibacterial peptide PR-39 induces chemotaxis of neutrophils, with a maximal response at 0.5-2 $\mu \mathrm{M}$, and stimulates extracellular $\mathrm{Ca}^{2+}$ influx in these cells, as well.
Neither lymphocytes nor peripheral blood monocytes or alveolar macrophages are attracted by PR-39 [24]. Interestingly, LL-37 causes almost complete inhibition of serum amyloid A (SAA)-induced neutrophil chemotaxis [25]. It seems to be very important to note down that cathelicidin LL-37 reduces neutrophil surface expression of CXCR2, but not CXCR1 [19, 26], i.e. the expression of receptor that mediates neutrophil migration to the sites of inflammation. The mechanism of CXCR2 down-regulation by LL-37 is due to the receptor internalization [19].

Some data indicated that cathelicidins directly activate neutrophils to mediator release. Human cathelicidin, at a concentration of $20 \mu \mathrm{g} / \mathrm{ml}$, stimulates neutrophils to the synthesis of pro-inflammatory CXCL8 under the control of mitogen-activated protein kinase (MAPK) p38 and extracellular signal-regulated kinase (ERK) [26, 27]. It is well known that this chemokine, acting via CXCR2, induces chemotaxis of not only neutrophils but also other granulocytes, and stimulates neutrophils to phagocytosis. On the other hand, interesting data suggest that LL-37 inhibits SAA-induced CXCL8 production and causes dramatic inhibition of ERK and p38 MAPK activities [25]. In turn, CXCL8 inhibition results in blocking production of acute phase proteins [25]. LL-37 activates neutrophils to the release of interleukin-1 receptor antagonist (IL-1Ra) the natural antagonist of IL-1 $\beta[26]$ and at the same time induces a dose-dependent increase in HNP-1, HNP-2, and HNP-3 $\alpha$-defensin gene expression [27]. What is more, extracellular release of these $\alpha$-defensins in response to LL-37 stimulation was observed [27]. Finally, LL-37 stimulates the generation of ROS in neutrophils [27-29] and this process is mediated by a flavoenzyme (most probably NADPH oxidase) and via increase in intracellular $\mathrm{Ca}^{2+}$ concentrations [27].

\section{Cathelicidins affect monocyte/macrophage activity}

Without any doubt, in the course of acute and chronic inflammation an important role is played by monocytes and macrophages. Given that these cell populations can produce both pro-inflammatory and anti-inflammatory mediators, it is obvious that they strongly direct the inflammatory processes and significantly influence an intensity of inflammation. Monocytes and macrophages have the ability to secrete a broad range of pro-inflammatory cytokines, such as IL-1 $\beta$, IL-6, IL-12, IL-18, IL-23, IL-27, and tumor necrosis factor (TNF). These cells also produce various chemokines (CCL2, CCL5, CCL7, CXCL1, CXCL2, CXCL8, CXCL9, CXCL10, CXCL11) as well as PGs, LTs, and platelet activating factor (PAF). Moreover, activated macrophages synthesize ROS and nitrogen intermediates that are highly toxic for microorganisms, but also induce tissue injury and lead to enhance inflammation. Finally, monocytes/macrophages are the 
source of various proteolytic enzymes that can act directly on cells and ECM proteins leading to tissue damage. On the other hand, there are a lot of monocyte/macrophage-derived mediators involved in suppressing inflammation, including some anti-inflammatory cytokines, i.e. IL-4, IL-10, IL-13, IL-19, and growth factors, i.e. transforming growth factor (TGF)- $\beta$ and vascular endothelial growth factor (VEGF). In fact, macrophages can exhibit pro- or anti-inflammatory properties and this is determined by stimuli from their local microenvironment. Thus, due to their cytokine/mediator profile two evidently distinct types of macrophages are designated. M1 macrophages, differentiated under the influence of granulocyte-macrophage colony-stimulating factor (GM-CSF), with a pro-inflammatory signature, and M2 macrophages, differentiated under the influence of macrophage colony-stimulating factor (M-CSF), with an anti-inflammatory signature [30, 31].

It is well established that human cathelicidin LL-37 activates FPR 2 molecules on monocytes, triggering monocytic $\beta_{1}$ - and $\beta_{2}$-integrin conformational change toward active conformation that allows for monocyte adhesion [32]. As in the case of neutrophils, LL-37 acts as potent chemoattractant for monocytes and can induce $\mathrm{Ca}^{2+}$ mobilization $[19,22]$. Moreover, murine CRAMP also causes monocyte migration in a dose-dependent manner and the peak response was observed at $400 \mathrm{nM}$. This CRAMP-induced monocyte chemotaxis seems to be mediated by a $G$ protein coupled receptor (GPCR), probably FPR2 molecule [23]. The chemotactic activity of bovine cathelicidin proBac7 toward monocytes is also documented [33]. It is interesting that murine CRAMP acts as a chemoattractant for monocyte-derived macrophages as well [23]. This cathelicidin induces ERK1/2 and p38 kinase phosphorylation and activation in monocytes, acting at a concentration as high as $50 \mu \mathrm{g} / \mathrm{ml}$. However, in the presence of GM-CSF, a cytokine found locally at sites of infection, LL-37 induces ERK1/2 phosphorylation at lower concentrations, such as $5-10 \mu \mathrm{g} / \mathrm{ml}$ [34].

More and more data indicate that cathelicidins by themselves directly stimulate monocytes to produce and release some cytokines and chemokines that may strongly modulate the course and intensity of inflammation. It was demonstrated that genes encoding chemokines essential for cell recruitment to the site of inflammation, such as CXCL1, CXCL8, CCL2, and CCL7 are significantly up-regulated in monocytes activated with LL-37 [34, 35]. On the contrary, LL-37 down-regulates CXCR2 expression on monocytes [19]. It is interesting that LL-37 does not enhance mRNA expression of pro-inflammatory cytokines TNF, IL-1 $\beta$, and IL-6 [34] and causes an increase in the transcription of the genes encoding anti-inflammatory cytokines such as IL-10 and IL-19 [35]. Human cathelicidin elicits modest up-regulation of CCL7 and IL-6 gene expression in peripheral blood mononuclear cells (PBMCs) as well [36]. Moreover, LL-37 alone activates monocytes to the production of CXCL8 and CCL2 $[34,35]$ and PBMCs to synthesis of CXCL8, CCL2, CCL5, and CCL7 [35, 36]. It was noticed, however, that this peptide stimulates the production of anti-inflammatory cytokine IL-1Ra but does not induce the synthesis of IL-10 in PBMCs [37].

Furthermore, there is information that cathelicidins influence monocyte responsiveness to some endogenous pro-inflammatory factors. Co-stimulation of PBMCs with LL-37 and IL-1 $\beta$ results in substantially augmented CCL7 and IL-6 mRNA accumulation and leads to an increase in the production of CCL2, CCL7, IL-6, and anti-inflammatory cytokine IL-10 [36]. LL-37 substantially enhances the production of CXCL8 and IL-6, but not TNF, in synergy with IL-1 $\beta$ [38]. In the synergistic production of CCL7 induced by LL-37 and IL-1 $\beta$, GPCR and phosphatidylinositol 3-kinase (PI3K), but not protein kinase (PK)C and PKA, a signaling pathway is involved [36]. LL-37 abrogates IL-32-induced TNF and IL- $1 \beta$ production by $97 \%$ in PBMCs and IL-32-induced IL-6 synthesis is suppressed by $50 \%$ in the presence of LL-37. In contrast, IL-32-mediated chemokine CXCL1 and CXCL8 synthesis is not altered in the presence of this peptide [37]. Moreover, LL-37 does not suppress IL-32-mediated production of anti-inflammatory IL-1Ra but inhibits IL-32-mediated IL-10 synthesis in PBMCs [37]. It is also documented that CCL2 and IL-6 production by PBMCs in response to GM-SCF stimulation is significantly enhanced in the presence of LL-37 [36]. Conversely, LL-37 has no effect on TNF-induced IL-1 $\beta$, IL-6, and CXCL8 production [38]. Interestingly, cathelicidin LL-37 strongly inhibits the production of TNF and IL-12 by monocytes stimulated with interferon (IFN)- $\gamma$ [39] and suppresses IFN- $\gamma$-induced CCL2 synthesis in PBMCs [36]. CCL2 production in response to LL-37 stimulation is inhibited in the presence of IL-4 or IL-12 [36].

Extremely intriguing are observations that LL-37 has a general anti-inflammatory effect on Toll-like receptor (TLR) stimulation, inhibiting pro-inflammatory cytokine release from monocytic cells stimulated with TLR2, TLR4, and TLR9 agonist. It was demonstrated that LL-37 peptide significantly inhibits the expression of specific pro-inflammatory genes up-regulated by nuclear factor (NF)- $\mathrm{B}$ in the presence of TLR4 ligand LPS. In contrast, LL-37 does not inhibit LPS-induced genes which antagonize inflammation and certain chemokine genes classically considered pro-inflammatory [38]. Moreover, LL-37, at very low concentrations $(\leq 1 \mu \mathrm{g} / \mathrm{ml})$, inhibits TNF synthesis and release from LPS-induced monocytes. This inhibitory effect increases to $\geq 95 \%$ with a dose of $20 \mu \mathrm{g} / \mathrm{ml}$ of LL-37. Similarly, LL-37 significantly inhibits TNF, IL-1 $\beta$, IL-6, and CXCL8 production in PBMCs stimulated with LPS [38]. It should pointed out, however, that LL-37 stimulation of LPS-primed monocytes leads to release of IL-1 $\beta$ acting via $\mathrm{P} 2 \mathrm{X}_{7}$ receptor [34, 40], and caspase-1 activation [40]. Furthermore, acting on PBMCs, LL-37 significantly decreases the production of IL-1 $\beta$, IL-6, TNF, and CXCL8 resulting 
from activation of TLR2 molecules by lipoteichoic acid (LTA) or synthetic tri-acylated lipopeptide Pam ${ }_{3}$ CSK4. In addition, LL-37 reduces to 50\%, CXCL8 secretion from PBMCs in response to stimulation with TLR9 agonist synthetic CpG oligodeoxynucleotide. In contrast, LL-37 enhances TNF and IL-6 production by CpG-stimulated PBMCs [38].

It is interesting that LL-37 very efficiently transports self-DNA into monocytes, leading to the production of type I IFNs in a TLR-independent manner [41]. There are some data that LL-37 can influence monocyte differentiation. Peptide LL-37 (re)directs M-CSF-driven differentiation of monocytes toward macrophages with a pro-inflammatory instead of an anti-inflammatory signature. These macrophages have a low expression of CD163, little of IL-10 and profound IL-12 production on LPS stimulation. LL-37 enhances the GM-CSF-driven macrophage differentiation as well. Additionally, it is proved that under the influence of LL-37 fully differentiated M2 macrophages produce less IL-10 and more IL-12 [42].

In like manner, cathelicidins can regulate the inflammatory activity of tissue macrophages. LL-37 directly up-regulates 29 genes in macrophages, including those encoding chemokine CCL7, anti-inflammatory cytokine IL-10, M-CSF, many chemokine receptors, and several metalloproteinases (MMPs). On the contrary, this peptide can down-regulate another 20 genes, including those encoding CCL3 and IL-12. The secretion of CCL2 in response to LL-37 stimulation is also observed [43]. There are intriguing data that peptide LL-37 can be an endogenous regulator of eicosanoid-dependent inflammatory responses since it promotes $\mathrm{LTB}_{4}$ and thromboxane (TX) $\mathrm{A}_{2}$ generation by macrophages. It should be stressed that LL-37-induced eicosanoid generation is time-resolved. This cathelicidin, acting via $\mathrm{P} 2 \mathrm{X}_{7}$ receptor, evokes $\mathrm{Ca}^{2+}$ mobilization, activation of ERK1/2 and p38 MAPKs, as well as cytosolic phospholipase $\mathrm{c}(\mathrm{PL}) \mathrm{A}_{2}$, and 5-lipoxygenase, leading to an early (1 hour) release of $\mathrm{LTB}_{4}$. Likewise, $\mathrm{TXA}_{2}$ production at early time involves signaling sequence LL-37 - P2X $\mathrm{X}_{7}$ receptor - cPLA 2 - cyclooxygenase-1 (COX-1) axis. Later on, 6-8 hours after LL-37-stimulation, internalized cathelicidin up-regulates COX-2 expression, promoting $\mathrm{TXA}_{2}$ production [44].

LL-37 significantly decreases Neisseria meningitidis endotoxin lipooligosaccharide (LOS)-induced both TNF and nitric oxide (NO) release from macrophages. In contrast, LL-37 and LOS are synergistic in enhancing ROS release from macrophages. This synergistic effect of LL-37 and TLR4 ligand LOS is a result of a direct effect exerted on the ROS generating enzymes mainly the NADPH oxidase complex [45]. What is more, the presence of LL-37 during the polarization of M1 macrophages by LPS and IFN- $\gamma$ results in strong inhibition of TNF and NO production and in a slight decrease in ROS synthesis [46]. Similarly, TNF synthesis by polarized M2 macro- phages in response to LPS stimulation is effectively inhibited by LL-37. This peptide has no effect on TNF production by macrophages activated with TLR2 synthetic ligand Pam ${ }_{3}$ CSK4 but dramatically inhibits LTA-induced TNF synthesis [46]. Scott et al. [43] demonstrated that LL-37 causes a substantial inhibition of not only LPS, but also LTA-, and arabinosylated lipoarabinomannan (AraLAM)-induced TNF synthesis by macrophages. It was also documented that murine cathelicidin CRAMP exerts a strong inhibition of LPS-, LTA-, and flagellin(TLR5 agonist) induced p38 and ERK phosphorylation in macrophages and strongly reduced TNF production by macrophages stimulated with LPS or LTA. However, murine CRAMP does not affect the production of IL-10 and chemokines CXCL1 and CCL3 by LPS-activated macrophages [47].

Kahlenberg et al. [48] observed that LL-37 activates caspase-1, the central enzyme of the inflammasome, in both human and murine macrophages, resulting in release of active IL-1 $\beta$ and IL-18. LL-37 activation the NLRP3 inflammasome utilizes $\mathrm{P} 2 \mathrm{X}_{7}$ receptor-mediated potassium efflux. On the other hand, it is worth noting that LL-37 inhibits LPS/ATP-induced IL- $1 \beta$ expression, caspase- 1 activation, inflammasome formation, as well as macrophage death by pyroptosis [49].

\section{Cathelicidins affect mast cell activity}

Mast cells are long-lived resident tissue cells widely distributed throughout connective tissues. These cells are particularly numerous beneath the subepithelial layers of the skin, in the airways, gastrointestinal and genitourinary tracts, and adjacent to blood vessels and nerves. They have the potential to secrete a wide spectrum of biologically active mediators, cytokines and chemokines. Upon activation, mast cells rapidly release preformed, cytoplasmic granule-associated mediators, such as histamine, neutral proteases (tryptase, chymase), proteoglycans, MMPs, as well as various preformed cytokines including IL-3, IL-4, IL-6, IL-10, TNF, and CXCL8. Moreover, in response to stimulation mast cells release various newly generated lipid mediators, including LTs, PGs, TXs, and PAF, and many cytokines and chemokines. These cells express numerous different receptors and thereby various endogenous and exogenous factors can activate mast cells to mediator/cytokine generation. Mast cell-derived mediators exert diverse effects on surrounding cells and tissues [50]. Therefore, mast cells are important players in homeostasis maintenance via involvement in angiogenesis, tissue remodeling and repair, and regulation of vascular permeability $[50,51]$. Moreover, they strongly influence both innate and acquired immune responses and are critical components of host defense against bacteria and viruses [52-54]. Mast cells take part in different pathological processes, including allergic disease as well $[50,51,55]$. Finally, in- 
asmuch as mast cell-derived mediators elicit pro-inflammatory, anti-inflammatory and immunoregulatory effects, these cells are essential in both promoting and limiting the inflammatory processes [52, 56, 57].

A few data indicated that LL-37 can exert direct effects on mast cells. It has been proven that this cathelicidin induces, in a dose-dependent fashion, degranulation of rat mature mast cells, as assessed by histamine or $\beta$-hexosaminidase release [58-60]. This is accompanied by intracellular $\mathrm{Ca}^{2+}$ mobilization [58]. Cathelicidin LL-37 promotes degranulation and preformed mediator release from human lung mast cells [61] and stimulates degranulation and $\mathrm{Ca}^{2+}$ mobilization in immature LAD2 human mast cells $[62,63]$ as well. Moreover, this peptide activates LAD2 mast cells to synthesis and releases the strong pro-inflammatory mediators such as $\mathrm{LTC}_{4}$ and $\mathrm{PGD}_{2}$ [64] and rat CRAMP stimulates mature mast cells to production and release cysteinyl (cys)LTs [65]. Importantly, anti-inflammatory cytokine IL-10, but not TNF, IL-6 and CCL5, strongly inhibits CRAMP-induced cysLT synthesis [65]. Moreover, LL-37 activates mast cells to produce newly generated cytokines, including IL-2, IL-4, IL6, IL-31, GM-CSF, and TNF, and chemokines CCL4 and CXCL8 $[60,63,64]$. This peptide, at a concentration of 20 $\mu \mathrm{g} / \mathrm{ml}$, induces IL-1 $\beta$, CCL2, and CCL3 mRNA increase in rat mast cells [60]. The cathelicidin CRAMP stimulates mast cells to TNF and CXCL8 release and induces GMCSF, IL-1 $\beta$, CCL2, and CCL3, but not IL-33, mRNA expression [65]. Yoshioka et al. [62] observed that LL-37, at a concentration of $1 \mu \mathrm{g} / \mathrm{ml}$, stimulates LAD2 mast cells to generate Th2 cytokines IL-4 and IL-5, but not IL-10, Th1 cytokine IL-2, but not IFN- $\gamma$ and TNF- $\beta$, pro-inflammatory cytokines TNF and IL-1 $\beta$, but not IL-6 and CXCL8. Moreover, LPS significantly reduced LL-37-induced IL-4 and IL5 , but not IL-1 $\beta$, generation. These intriguing findings suggest that LL-37 co-existing with the bacterial components may change the mast cell function toward innate immunity. There are also very interesting data that cathelicidins LL-37 and CRAMP act as potent mast cell chemoattractants [60, $65,66]$, and thereby cathelicidins may additionally enhance inflammatory response via attracting mast cells to pathogen entry site. Besides, it was observed that LL-37, at a concentration of $1 \mu \mathrm{g} / \mathrm{ml}$, up-regulates both TLR 4 mRNA and TLR4 protein expression on LAD2 mast cells, and in that way, cathelicidin enhances mast cell capability to detect invading pathogen [62].

Up to date, the mechanisms by which cathelicidins activate mast cells are not fully clarified. Some data indicate that GPCRs mediate LL-37-induced signal transduction pathway in mast cells $[58,66]$. There are some observations that LL-37 acts via Mas-related gene (Mrg) $\mathrm{X} 2$ receptor [63] or through FPR2 and receptor $\mathrm{P} 2 \mathrm{X}_{7}$ [62]. Moreover, it was suggested that LL-37 activates mast cells in PLC-dependent manner $[58,66]$. In addition, some data indicate that in LL-37-dependent mast cell activation PI3K and MAPK signaling pathways are involved [59, 60, 62, 64, 65].

\section{Cathelicidins affect other inflammatory cell activity}

Neutrophils, monocytes, macrophages, and mast cells undoubtedly play a key role in inflammation. However, it is not to overestimate the role of other populations of host cells in inflammatory processes. Limited data show that human cathelicidin LL-37 and mouse CRAMP can modulate activity of eosinophils, dendritic cells, epithelial cells, and keratinocytes.

It is documented that LL-37, at a concentration range from 15 to $30 \mu \mathrm{g} / \mathrm{ml}$, directly stimulates eosinophils to cysLTs and $\mathrm{LTB}_{4}$ release and priming with eosinophilopoietic cytokine GM-CSF or IL-5 significantly enhances LL-37induced cysLT secretion. Moreover, in response to LL-37 activation, eosinophils release eosinophil cationic protein (ECP). Cathelicidin-mediated cysLT release is dependent on FPR2 molecule. It is also indicated that LL-37 enhances cytosolic PLA $\mathrm{P}_{2}$ activity, triggers intracellular translocation of 5-lipoxygenase and $\mathrm{LTC}_{4}$ synthase to perinuclear localization of lipid bodies [67]. Human cathelicidin LL-37, at a high concentration of $40 \mu \mathrm{g} / \mathrm{ml}$, induces eosinophil chemotaxis and this effect is mediated via FPR2 molecules [21]. In the course of inflammatory processes dendritic cells take part to some extent [68], however the influence of LL-37 on dendritic cell functioning is currently almost unknown. It was indicated that human cathelicidin LL-37 strongly inhibits LPS-mediated pro-inflammatory cytokine, i.e. IL-6 and TNF, generation [35, 69-71]. Similarly, murine CRAMP causes a decrease in IL-6, but not IL-1 $\beta$, synthesis by dendritic cells in response to LPS stimulation [71]. LL-37 also blocks LPS-induced chemokine CXCL8 synthesis $[35,71]$ and declines LPS-induced up-regulation of CXCR7 [69]. Human cathelicidin LL-37 completely inhibits IL-6 and TNF generation in response to LTA and flagellin activation as well [69]. Worthy of note is that LL-37 significantly abrogates LPS-, LTA-, and flagellin-mediated production of IL-12 [69, 70], a pro-inflammatory cytokine with immunoregulatory functions which promotes differentiation of T cells into Th1 cells. Di Nardo et al. [71] observed that pretreatment of monocyte-derived dendritic cells with LL-37 significantly reduces LPS induction of IL-10. On the contrary, Mookherjee et al. [35] established that this peptide enhances LPS-mediated IL-10 production by myeloid dendritic cells. It was also documented that LL-37 inhibits IFN- $\gamma$-induced TNF synthesis by dendritic cells [70].

Only few data indicate that cathelicidin LL-37 may affect epithelial cell activity. It was established that this peptide stimulates airway epithelial cell migration and proliferation and this effect is mediated through GPCR and epidermal growth factor receptor (EGFR) [72]. Pistolic et 
al. [73] established that this peptide by itself induces IL6, CXCL1, and CXCL8 release from bronchial epithelial cells. Likewise, Tjabringa et al. [74] indicated that LL-37, at concentrations as high as $50-100 \mu \mathrm{g} / \mathrm{ml}$, stimulates airway epithelial cells by activation of ERK1/2 and increased release of CXCL8. It is interesting that LL-37 activates epithelial cells by transactivation of the EGFR via metalloproteinase-dependent processing of EGFR ligands. Furthermore, this host defense peptide augments TLR5-mediated (flagellin) production of IL-6 by epithelial cells [73]. Significantly higher levels of CXCL8 and IL-6 are secreted by lung epithelial cells after stimulation with TLR4 agonist LPS in the presence of LL-37 as well [75]. Filewod et al. [76] showed that LL-37, at concentrations of 2-3 $\mu \mathrm{g} / \mathrm{ml}$, alters bronchial epithelial cell responses to pro-inflammatory stimuli. In combination with IL-1 $\beta$, TLR5 agonist flagellin, or TLR3 agonist polyinosinic:polycytidylic acid (polyI:C) LL-37 causes synergistically an increase in CXCL8 production and in combination with TLR2 agonist $\mathrm{Pam}_{3} \mathrm{C}$ SK4 this cathelicidin synergistically induces transcription and release of both IL-6 and CXCL8 from these cells.

There are limited data on LL-37 influence on the keratinocyte activity. It was indicated that this human cathelicidin induces keratinocyte migration and proliferation. The effect of LL-37 occurs via heparin-binding epidermal growth factor (HB-EGF)-mediated EGFR transactivation and signal transducer and activator of transcription (STAT)1, and STAT3 intracellular molecules are involved $[77,78]$. What is more, LL-37 by itself stimulates keratinocytes to synthesis and release of different pro-inflamma- tory and/or immunoregulatory cytokines, including IL-6, IL-18, IL-20, and GM-CSF [77, 79, 80]. In response to LL-37 activation, keratinocytes produce some chemokines, i.e. CCL2, CCL5, CCL20, CXCL8, and CXCL10 [77, 79, 80]. It is important to note that human cathelicidin stimulates the synthesis of anti-inflammatory cytokine IL-10 in keratinocytes [77] and significantly up-regulates gene encoding COX2 [80] as well. Cathelicidin peptide-induced cytokine/chemokine production in keratinocytes involves EGFR, G protein, and PLC signaling pathways $[77,80]$. LL-37-induced IL-18 secretion is probably via caspase-1-independent pathway and through p39 and ERK1/2 MAPK pathways [79]. Very intriguing are observations that $\beta$-defensins HBD- $1,-2,-3$, and -4 have a synergistic effect on IL-18 secretion by keratinocytes in response to LL-37 stimulation [79]. It should be also noted that LL-37 in combination with IL-1 $\beta$ or TLR5 agonist synergistically increases CXCL8 synthesis by both proliferating and differentiated keratinocytes [76].

\section{Concluding remarks}

Cathelicidins, like other AMPs, exhibit antimicrobial activities against a broad spectrum of microbes, including both Gram-positive and Gram-negative bacteria, enveloped viruses, and fungi. These peptides directly kill the invaded microorganisms by perturbing their cell membranes and can neutralize biological activities of endotoxin. Nowadays, more and more data indicate that cathelicidins, in addition to their antimicrobial properties, have the potential

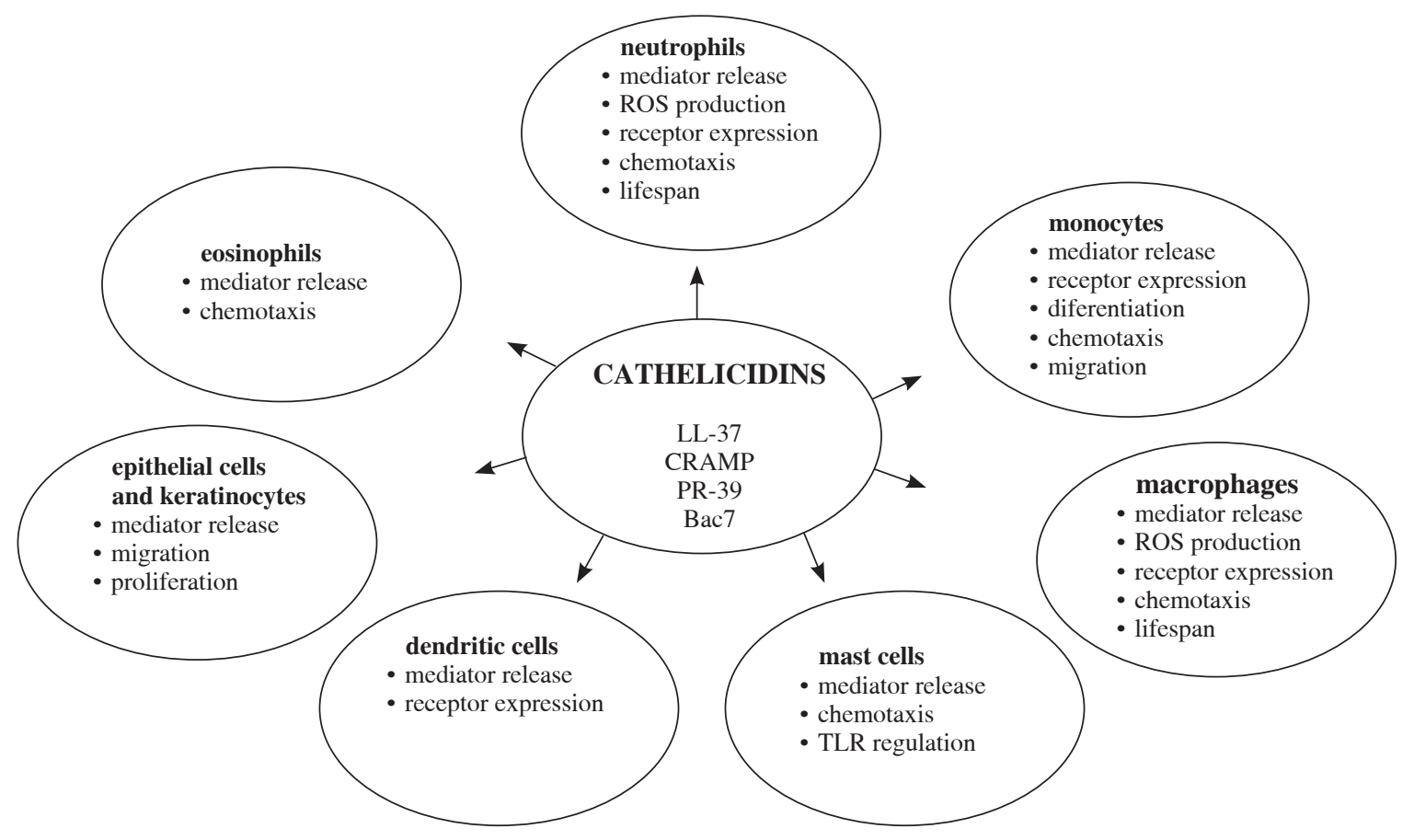

Fig. 1. Immunomodulatory activities of cathelicidins 
to influence and modulate, both directly and indirectly, the activity of various cell populations involved in inflammatory processes (Fig. 1). First of all, it should be underlined that cathelicidins may intensify the course of inflammation via attracting neutrophils, monocytes, macrophages, eosinophils, and mast cells to pathogen entry site. Importantly, recruiting cells, and especially neutrophils, are a rich source of cathelicidins. Thus, high concentrations of these peptides can be most easily achieved at the sites of immune cell accumulation. It is worthy of note, however, that cathelicidin LL-37 reduces CXCR2 expression on neutrophils and monocytes, i.e. the receptor that mediates chemokine-induced cell migration. Cathelicidins also stimulate migration and proliferation of epithelial cells and keratinocytes. Moreover, these peptides may exacerbate inflammatory reactions by suppressing apoptosis and prolonging the lifespan of neutrophils. In addition, cathelicidin LL-37 suppresses caspase-1 dependent pyroptosis in macrophages.

Cathelicidins directly activate different cell populations to production and release of different pro-inflammatory and immunoregulatory cytokines, inter alia IL-1 $\beta$, IL-6, IL-18, IL-20, TNF, and GM-CSF. Likewise, these peptides induce the production of various chemokines, including CCL2, CCL5, CCL7, CCL20, CXCL1, CXCL4, CXCL8, and CXCL10. Furthermore, in response to cathelicidin activation mast cells, macrophages, and eosinophils generate potent pro-inflammatory mediators, such as cysLTs, $\mathrm{LTB}_{4}$, $\mathrm{TXA}_{2}, \mathrm{PGD}_{2}$ and histamine. It is crucial that cathelicidins stimulate production and release of ROS, NO, ECP, and some proteinases, which take part in tissue damage. On the other hand, cathelicidins can induce the synthesis of some anti-inflammatory mediators; macrophages and keratinocytes produce IL-10, monocytes synthesize IL-10 and IL-19, and monocytes and neutrophils generate IL-1Ra.

Without any doubt it is intriguing that cathelicidins can affect activity of various cell types indirectly. Peptide LL-37 acting in synergy with IL-1 $\beta$ causes a significant increase in CXCL8 production by epithelial cells and keratinocytes and in not only CCL2, CCL7, IL-6, but also IL-10, by monocytes. This cathelicidin exerts synergistic effect on GM-CSF-induced CCL2 and IL-6 synthesis by monocytes and significantly enhances IL-18 secretion from keratinocytes stimulated with $\beta$-defensins. Otherwise, co-stimulation of monocytes and dendritic cells with LL-37 and IFN- $\gamma$ causes an influential decrease in TNF synthesis, and monocyte co-stimulation with LL-37 and IL-32 results in a decrease in TNF, IL-6, IL-1 $\beta$, and also IL-10 generation. In addition, cathelicidins also appear to have functions in modulating TLR-mediated cell responses. These peptides strongly inhibit pro-inflammatory cytokine release, i.e. TNF, IL-1 $\beta$, IL-6 and chemokine CXCL8, from monocytes stimulated with TLR2 or TLR4 agonists. It is important to stress that LL-37 significantly decreases CXCL8 production by monocytes activated with TLR9 ligand but enhances TNF and IL-6 synthesis. Similarly, cathelicidins strongly decrease TLR2- and TLR4-mediated TNF and NO synthesis in macrophages, but enhance ROS release in response to TLR4 stimulation. Furthermore, cathelicidins strongly inhibit IL-6, IL-12, TNF, and CXCL8 generation by dendritic cells stimulated by TLR2, TLR4, or TLR5 ligands. On the contrary, it is very interesting that synergistic action of LL-37 and TLR2, TLR3, TLR4, or TLR5 ligands induces an increase in IL-6 and CXCL8 generation by epithelial cells and keratinocytes.

To date, it has not been entirely clear what the exact cathelicidin concentrations in physiological or pathophysiological conditions and in inflammatory milieu are. Nevertheless, there are data that in healthy individuals the LL-37 levels were as low as $39.9 \mathrm{pg} / \mathrm{ml}$ in sputum [81], $1-4 \mathrm{ng} / \mathrm{ml}$ in tracheal aspirate samples obtained from new-born children [82], and 1.1 and $1.7 \mathrm{ng} / \mathrm{ml}$ in plasma of neonatal and maternal blood, respectively [83, 84]. Some information indicates that in healthy individuals the concentrations of LL-37 are about $27.2 \mathrm{ng} / \mathrm{ml}$ in plasma [85] and $30.5 \mathrm{ng} / \mathrm{ml}$ in saliva [86]. What is more, concentrations of LL-37 in sweat and bronchoalveolar lavage fluid (BAL) of healthy infants are significantly higher and amount up to $4.47 \mu \mathrm{g} /$ $\mathrm{ml}$ [87] and $4.8 \mu \mathrm{g} / \mathrm{ml}$, respectively [88]. Interestingly, the levels of LL-37 in sperm are as high as $86.5 \mu \mathrm{g} / \mathrm{ml}$ [89]. In pathological conditions, the concentrations of this cathelicidin are highly differentiated. In sputum obtained from patients suffering from cystic fibrosis $(\mathrm{CF})$, chronic obstructive pulmonary disease (COPD), and asthma, LL-37 concentrations were as low as $79.6 \mathrm{pg} / \mathrm{ml}, 75.3 \mathrm{pg} / \mathrm{ml}$, and $13.6 \mathrm{pg} / \mathrm{ml}$, respectively [81]. Moreover, the concentration of LL-37 in plasma of patients with sepsis was $13.7 \mathrm{ng} / \mathrm{ml}$ [85], and $22.5 \mathrm{ng} / \mathrm{ml}$ in saliva of individuals suffering from chronic periodontitis [86]. Chen et al. [90] determined that concentrations of LL-37 in BAL of patients with CF were from an undetectable level up to $15 \mu \mathrm{g} / \mathrm{ml}$. In turn, the level of this peptide was up to even $1.4 \mathrm{mg} / \mathrm{ml}$ in skin-biopsy samples from patients with psoriasis [91]. The cathelicidin effective concentrations used in the in vitro studies were in the range between $0.01 \mu \mathrm{g} / \mathrm{ml}$ and $50 \mu \mathrm{g} / \mathrm{ml}$. Thus, it can be assumed that the cathelicidin concentrations used in experiments in vitro correspond to those occurring in vivo in different pathophysiological conditions, and especially during infection and inflammation in the local milieu.

In conclusion, it is now obvious that cathelicidins have various immunomodulatory activities. Therefore, these peptides should be considered as the multifunctional molecules that strongly affect the course of inflammation. It is important to state, however, that the exact role of these peptides in inflammation is ambiguous, as cathelicidins might promote or reduce the inflammatory intensity. Moreover, without any doubt these host derived peptides affect host defense against microorganisms by modulating epithelial responses to infecting pathogens, by balancing the TLR-mediated cell response to bacteria and viruses, and by influencing the inflammatory processes at sites of patho- 
gen entry. Without any doubt, further studies are necessary to fully clarify the exact role of cathelicidins in inflammation and innate immunity developed during infection.

This work was supported by the Medical University of Łódź (Grant No. 503/6-164-01/503-1).

The authors declare no conflict of interest.

\section{References}

1. Choi K-Y, Chow LNY, Mookherjee N (2012): Cationic host defence peptides: multifaceted role in immune modulation and inflammation. J Innate Immun 4: 361-370.

2. Barlow PG, Findlay EG, Currie SM, Davidson DJ (2014): Antiviral potential of cathelicidins. Future Microbiol 9: 55-73.

3. Mansour SC, Pena OM, Hancock RE (2014): Host defense peptides: front-line immunomodulators. Trends Immun 35: 443-450.

4. Guani-Guerra E, Santos-Mendoza T, Lugo-Reyes SO, Téra LM (2010): Antimicrobial peptides: general overview and clinical implications in human health and disease. Clin Immunol 135: 1-11

5. Zanetti M (2004): Cathelicidins, multifunctional peptides of the innate immunity. J Leukoc Biol 75: 39-38.

6. Tomasinsig L, Zanetti M (2005): The cathelicidins - structure, function and evolution. Curr Protein Pept Sci 6: 23-34.

7. Vandamme D, Landuyt B, Luyten W, Schoofs L (2012) A comprehensive summary of LL-37, the factotum human cathelicidin peptide. Cell Immunol 280: 22-35.

8. Wong JH, Ng TB, Legowska A, et al (2011): Antifungal action of human cathelicidin fragment (LL13-37) on Candida albicans. Peptides 32: 1996-2002.

9. Bocchinfuso G, Palleschi A, Orioni B, et al (2009): Different mechanisms of action of antimicrobial peptides: insights from fluorescence spectroscopy experiments and molecular dynamics simulations. J Pept Sci 15: 550-558.

10. Park Y, Hahm KS (2005): Antimicrobial peptides (AMPs): peptide structure and mode of action. J Biochem Mol Biol 38: 507-516.

11. Dürr UHN, Sudheendra US, Ramamoorthy A (2006): LL-37, the only human member of the cathelicidin family of antimicrobial peptides. Biochim Biophys Acta 1758: 1408-1425.

12. Mantovani A, Cassatella MA, Costantini C, Jaillon S (2011): Neutrophils in the activation and regulation of innate and adaptive immunity. Nat Rev Immunol 11: 519-531.

13. Wright HL, Moots RJ, Bucknall RC, Edwards SW (2010): Neutrophil function in inflammation and inflammatory diseases. Rheumatology (Oxford) 49: 1618-1631.

14. Jaillon S, Galdiero MR, Del Prete D, et al. (2013): Neutrophils in innate and adaptive immunity. Semin Immunopathol 35: 377-394.

15. Kolaczkowska E, Kubes P (2013): Neutrophil recruitment and function in health and inflammation. Nat Rev Immunol 13: 159-175.

16. Nagaoka I, Tamura H, Hirata M (2006): An antimicrobial cathelicidin peptide, human CAP18/LL-37, suppresses neutrophil apoptosis via the activation of formyl-peptide receptor-like 1 and P2X7. J Immunol 176: 3044-3052.

17. Nagaoka I, Suzuki K, Niyonsaba F, et al. (2012): Modulation of neutrophil apoptosis by antimicrobial peptides. ISRN Microbiol 2012: 2012: 345791 .
18. Barlow PG, Li Y, Wilkinson TS, et al. (2006): The human cationic host defense peptide LL-37 mediates contrasting effects on apoptotic pathways in different primary cells of the innate immune system. J Leukoc Biol 80: 509-520.

19. Zhang Z, Cherryholmes G, Chang F, et al. (2009): Evidence that cathelicidin peptide LL-37 may act as a functional ligand for CXCR2 on human neutrophils. Eur J Immunol 39: 31813194.

20. Agerberth B, Charo J, Werr J, et al. (2000): The human antimicrobial and chemotactic peptides LL-37 and $\alpha$-defensins are expressed by specific lymphocyte and monocyte populations. Blood 96: 3086-3093.

21. Tjabringa GS, Ninaber DK, Drijfhout JW, et al. (2006): Human cathelicidin LL-37 is a chemoattractant for eosinophils and neutrophils that acts via formyl-peptide receptors. Int Arch Allergy Immunol 140: 103-112.

22. De Yang, Chen Q, Schmidt AP, et al. (2000): LL-37, the neutrophil granule- and epithelial cell-derived cathelicidin, utilizes formyl peptide receptor-like 1 (Fprl1) as a receptor to chemoattract human peripheral blood neutrophils, monocytes, and T cells. J Exp Med 192: 1069-1074.

23. Kurosaka K, Chen Q, Yarovinsky F, et al. (2005): Mouse cathelin-related antimicrobial peptide chemoattracts leukocytes using formyl peptide receptor-like 1/mouse formyl peptide receptor-like 2 as the receptor and acts as an immune adjuvant. J Immunol 174: 6257-6265.

24. Huang HJ, Ross CR, Blecha F (1997): Chemoattractant properties of PR-39, a neutrophil antibacterial peptide. J Leukoc Biol 61: 624-629.

25. Lee HY, Kim SD, Shim JW, et al. (2009): LL-37 inhibits serum amyloid A-induced IL-8 production in human neutrophils. Exp Mol Med 41: 325-333.

26. Zhang Z, Cherryholmes G, Shively JE (2008): Neutrophil secondary necrosis is induced by LL-37 derived from cathelicidin. J Leukoc Biol 84: 780-788.

27. Zheng Y, Niyonsaba F, Ushio H, et al. (2007): Cathelicidin LL-37 induces the generation of reactive oxygen species and release of human $\alpha$-defensins from neutrophils. Br J Dermatol 157: 1124-1131.

28. Tripathi S, Verma A, Kim EJ, et al. (2014): LL-37 modulates human neutrophil responses to influenza A virus. J Leukoc Biol 96: 931-938.

29. Alalwani SM, Sierigk J, Herr C, et al. (2010): The antimicrobial peptide LL-37 modulates the inflammatory and host defense response of human neutrophils. Eur J Immunol 40: 1118-1126.

30. Laskin DL, Sunil VR, Gardner CR, Laskin JD (2011): Macrophages and tissue injury: agents of defense or destruction? Annu Rev Pharmacol Toxicol 51: 267-288.

31. Yona S, Jung S (2010): Monocytes: subsets, origins, fates and functions. Curr Opin Hematol 17: 53-59.

32. Wantha S, Alard JE, Megens RT, et al. (2013): Neutrophil-derived cathelicidin promotes adhesion of classical monocytes. Circ Res 112: 792-801.

33. Verbanac D, Zanetti M, Romeo D (1993): Chemotactic and protease-inhibiting activities of antibiotic peptide precursors. FEBS Lett 317: 255-258.

34. Bowdish DM, Davidson DJ, Speert DP, Hancock RE (2004): The human cationic peptide LL-37 induces activation of the extracellular signal-regulated kinase and p38 kinase pathways in primary human monocytes. J Immunol 172: 3758-3765.

35. Mookherjee N, Hamill P, Gardy J, et al. (2009): Systems biology evaluation of immune responses induced by human host 
defence peptide LL-37 in mononuclear cells. Mol Biosyst 5: 483-496.

36. Yu J, Mookherjee N, Wee K, et al. (2007): Host defense peptide LL-37, in synergy with inflammatory mediator IL-1 $\beta$, augments immune responses by multiple pathways. J Immunol 179: 7684-7691.

37. Choi KY, Napper S, Mookherjee N (2014): Human cathelicidin LL-37 and its derivative IG-19 regulate interleukin-32-induced inflammation. Immunology 143: 68-80.

38. Mookherjee N, Brown KL, Bowdish DM, et al. (2006): Modulation of the TLR-mediated inflammatory response by the endogenous human host defense peptide LL-37. J Immunol 176: 2455-2464.

39. Nijnik A, Pistolic J, Wyatt A, et al. (2009): Human cathelicidin peptide LL-37 modulates the effects of IFN- $\gamma$ on APCs. J Immunol 183:5788-5798.

40. Elssner A, Duncan M, Gavrilin M, Wewers MD (2004): A novel P2X7 receptor activator, the human cathelicidin-derived peptide LL37, induces IL-1 $\beta$ processing and release. J Immunol 172: 4987-4994.

41. Chamilos G, Gregorio J, Meller S, et al. (2012): Cytosolic sensing of extracellular self-DNA transported into monocytes by the antimicrobial peptide LL37. Blood 120: 3699-3707.

42. van der Does AM, Beekhuizen H, Ravensbergen B, et al. (2010): LL-37 directs macrophage differentiation toward macrophages with a proinflammatory signature. J Immunol 185: 1442-1449.

43. Scott MG, Davidson DJ, Gold MR, et al. (2002): The human antimicrobial peptide LL-37 is a multifunctional modulator of innate immune responses. J Immunol 169: 3883-3891.

44. Wan M, Soehnlein O, Tang X, et al. (2014): Cathelicidin LL37 induces time-resolved release of LTB4 and TXA2 by human macrophages and triggers eicosanoid generation in vivo. FASEB J 28: 3456-3467.

45. Zughaier SM, Shafer WM, Stephens DS (2005): Antimicrobial peptides and endotoxin inhibit cytokine and nitric oxide release but amplify respiratory burst response in human and murine macrophages. Cell Microbiol 7: 1251-1262.

46. Brown KL, Poon GF, Birkenhead D, et al. (2011): Host defense peptide LL-37 selectively reduces proinflammatory macrophage responses. J Immunol 186: 5497-5505.

47. Pinheiro da Silva F, Gallo RL, Nizet V (2009): Differing effects of exogenous or endogenous cathelicidin on macrophage toll-like receptor signaling. Immunol Cell Biol 87: 496-500.

48. Kahlenberg JM, Carmona-Rivera C, Smith CK, Kaplan MJ (2013): Neutrophil extracellular trap-associated protein activation of the NLRP3 inflammasome is enhanced in lupus macrophages. J Immunol 190: 1217-1226.

49. Hu Z, Murakami T, Suzuki K, et al. (2014): Antimicrobial cathelicidin peptide LL-37 inhibits the LPS/ATP-induced pyroptosis of macrophages by dual mechanism. PloS One 9: e85765.

50. Rao KN, Brown MA (2008): Mast cells: multifaceted immune cells with diverse roles in health and disease. Ann N Y Acad Sci 1143: 83-104.

51. Rodewald HR, Feyerabend TB (2012): Widespread immunological functions of mast cells: fact or fiction? Immunity 37: 13-24.

52. Galli SJ, Grimbaldeston M, Tsai M (2008): Immunomodulatory mast cells: negative, as well as positive, regulators of immunity. Nat Rev Immunol 8: 478-486.

53. Witczak P, Brzezińska-Błaszczyk E (2012): Mast cells in viral infections. Postepy Hig Med Dosw 66: 231-244.
54. Trivedi NH, Guentzel MN, Rodriguez AR, et al. (2013): Mast cells: multitalented facilitators of protection against bacterial pathogens. Expert Rev Clin Immunol 9: 129-138.

55. Amin K (2012): The role of mast cells in allergic inflammation. Respir Med 106: 9-14.

56. Theoharides TC, Alysandratos KD, Angelidou A, et al. (2012): Mast cells and inflammation. Biochim Biophys Acta. 1822: 21-33.

57. Metz M, Grimbaldeston MA, Nakae S, et al. (2007): Mast cells in the promotion and limitation of chronic inflammation. Immunol Rev 217: 304-328.

58. Niyonsaba F, Someya A, Hirata M, et al. (2001): Evaluation of the effects of peptide antibiotics human $\beta$-defensins-1/-2 and LL-37 on histamine release and prostaglandin D2 production from mast cells. Eur J Immunol 31: 1066-1075.

59. Chen X, Niyonsaba F, Ushio H, et al. (2006): Human cathelicidin LL-37 increases vascular permeability in the skin via mast cell activation, and phosphorylates MAP kinases p38 and ERK in mast cells. J Dermatol Sci 43: 63-66.

60. Bąbolewska E, Brzezińska-Błaszczyk E (2015): Human-derived cathelicidin LL-37 directly activates mast cells to proinflammatory mediator synthesis and migratory response. Cell Immunol 293: 67-73.

61. Schiemann F, Brandt E, Gross R, et al. (2009): The cathelicidin LL-37 activates human mast cells and is degraded by mast cell tryptase: counter-regulation by CXCL4. J Immunol 183: 2223-2231.

62. Yoshioka M, Fukuishi N, Kubo Y, et al. (2008): Human cathelicidin CAP18/LL-37 changes mast cell function toward innate immunity. Biol Pharm Bull 31: 212-216.

63. Subramanian H, Gupta K, Guo Q, et al. (2011): Mas-related gene $\mathrm{X} 2(\mathrm{MrgX} 2)$ is a novel G protein-coupled receptor for the antimicrobial peptide LL-37 in human mast cells: resistance to receptor phosphorylation, desensitization, and internalization. J Biol Chem 286: 44739-44749.

64. Niyonsaba F, Ushio H, Hara M, et al. (2010): Antimicrobial peptides human $\beta$-defensins and cathelicidin LL-37 induce the secretion of a pruritogenic cytokine IL-31 by human mast cells. J Immunol 184: 3526-3534.

65. Bąbolewska E, Pietrzak A, Brzezińska-Błaszczyk E (2014): Cathelicidin rCRAMP stimulates rat mast cells to generate cysteinyl leukotrienes, synthesize TNF and migrate: involvement of PLC/A2, PI3K and MAPK signaling pathways. Int Immunol 26: 637-646.

66. Niyonsaba F, Iwabuchi K, Someya A, et al. (2002): A cathelicidin family of human antibacterial peptide LL-37 induces mast cell chemotaxis. Immunology 106: 20-26.

67. Sun J, Dahlén B, Agerberth B, Haeggström JZ (2013): The antimicrobial peptide LL-37 induces synthesis and release of cysteinyl leukotrienes from human eosinophils-implications for asthma. Allergy 68: 304-311.

68. Segura E, Amigorena S (2013): Inflammatory dendritic cells in mice and humans. Trends Immunol 34: 440-445.

69. Kandler K, Shaykhiev R, Kleemann P, et al. (2006): The anti-microbial peptide LL-37 inhibits the activation of dendritic cells by TLR ligands. Int Immunol 18: 1729-1736.

70. Nijnik A, Pistolic J, Wyatt A, et al. (2009): Human cathelicidin peptide LL-37 modulates the effects of IFN- $\gamma$ on APCs. J Immunol 183: 5788-5798.

71. Di Nardo A, Braff MH, Taylor KR, et al. (2007): Cathelicidin antimicrobial peptides block dendritic cell TLR4 activation and allergic contact sensitization. J Immunol 178: 1829-1834. 
72. Shaykhiev R, Beisswenger C, Kändler K, et al. (2005): Human endogenous antibiotic LL-37 stimulates airway epithelial cell proliferation and wound closure. Am J Physiol Lung Cell Mol Physiol 289: L842-884.

73. Pistolic J, Cosseau C, Li Y, et al. (2009): Host defence peptide LL-37 induces IL-6 expression in human bronchial epithelial cells by activation of the NF- $\mathrm{KB}$ signaling pathway. J Innate Immun 1: 254-267.

74. Tjabringa GS, Aarbiou J, Ninaber DK, et al. (2003): The antimicrobial peptide LL-37 activates innate immunity at the airway epithelial surface by transactivation of the epidermal growth factor receptor. J Immunol 171: 6690-6696.

75. Shaykhiev R, Sierigk J, Herr C, et al. (2010): The antimicrobial peptide cathelicidin enhances activation of lung epithelial cells by LPS. FASEB J 24: 4756-4766.

76. Filewod NC, Pistolic J, Hancock RE (2009): Low concentrations of LL-37 alter IL-8 production by keratinocytes and bronchial epithelial cells in response to proinflammatory stimuli. FEMS Immunol Med Microbiol 56: 233-240.

77. Niyonsaba F, Ushio H, Nakano N, et al. (2007): Antimicrobial peptides human $\beta$-defensins stimulate epidermal keratinocyte migration, proliferation and production of proinflammatory cytokines and chemokines. J Invest Dermatol 127: 594-604.

78. Tokumaru S, Sayama K, Shirakata Y, et al. (2005): Induction of keratinocyte migration via transactivation of the epidermal growth factor receptor by the antimicrobial peptide LL-37. J Immunol 175: 4662-4668.

79. Niyonsaba F, Ushio H, Nagaoka I, et al. (2005): The human $\beta$-defensins $(-1,-2,-3,-4)$ and cathelicidin LL-37 induce IL-18 secretion through p38 and ERK MAPK activation in primary human keratinocytes. J Immunol 175: 1776-1784.

80. Braff MH, Hawkins MA, Di Nardo A, et al. (2005): Structure-function relationships among human cathelicidin peptides: dissociation of antimicrobial properties from host immunostimulatory activities. J Immunol 174: 4271-4278.

81. Xiao A, Hsu YP, Ishizaka A, et al. (2005): Sputum cathelicidin, urokinase plasminogen activation system components, and cytokines discriminate cystic fibrosis, COPD, and asthma inflammation. Chest 128: 2316-2326.

82. Starner TD,Agerberth B,Gudmundsson GH, McCray PB Jr (2005): Expression and activity of $\beta$-defensins and LL-37 in the developing human lung. J Immunol 174: 1608-1615.

83. Mandic Havelka A, Yektaei-Karin E, Hultenby K, et al. (2010): Maternal plasma level of antimicrobial peptide LL37 is a major determinant factor of neonatal plasma LL37 level. Acta Paediatr 99: 836-841.

84. Srrrensen O, Cowland JB, Askaa J, Borregaard N (1997): An ELISA for hCAP-18, the cathelicidin present in human neutrophils and plasma. J Immunol Methods 206: 53-59.

85. Jeng L, Yamshchikov AV, Judd SE, et al. (2009): Alterations in vitamin $\mathrm{D}$ status and anti-microbial peptide levels in patients in the intensive care unit with sepsis. J Transl Med 7: 28.

86. Davidopoulou S, Diza E, Sakellari D, et al. (2013): Salivary concentration of free LL-37 in edentulism, chronic periodontitis and healthy periodontium. Arch Oral Biol 58: 930-934.

87. Murakami M, Ohtake T, Dorschner RA, et al. (2002): Cathelicidin anti-microbial peptide expression in sweat, an innate defense system for the skin. J Invest Dermatol 119: 1090-1095.

88. Schaller-Bals S, Schulze A, Bals R (2002): Increased levels of antimicrobial peptides in tracheal aspirates of newborn infants during infection. Am J Respir Crit Care Med 165: 992-995.

89. Malm J, Srrrensen O, Persson T, et al. (2002): The human cationic antimicrobial protein (hCAP-18) is expressed in the epithelium of human epididymis, is present in seminal plasma at high concentrations, and is attached to spermatozoa. Infect Immun 68: 4297-4302.

90. Chen CI, Schaller-Bals S, Paul KP, et al. (2004): $\beta$-defensins and LL-37 in bronchoalveolar lavage fluid of patients with cystic fibrosis. J Cyst Fibro 3: 45-50.

91. Ong PY, Ohtake T, Brandt C, et al. (2002): Endogenous antimicrobial peptides and skin infections in atopic dermatitis. N Engl J Med 347: 1151-1160. 